\title{
Research on rotate status of non-circular cross-section discrete rods
}

\author{
Junting Yin ${ }^{1, a}$, Gang $\mathrm{Li}^{2, \mathrm{~b}}$ \\ ${ }^{1}$ Xi'an Modern Chemistry Research Institute, Xi'an 710065, China \\ a1371965619@qq.com @163.com, bnpzk140@163.com
}

Keywords: warhead, discrete rod, non-circular cross-section, rotate, numerical simulation.

\begin{abstract}
Put forward a circumferential configuration non-circular cross-section discrete rod and it's charge structure. The results of numerical simulation show, by adjusting discrete rod and matching detonation loading, the charge circumferential integration of the discrete rod can dispersing along the radial direction and rotating simultaneously, possess a higher penetration ability to thin-thickness target, also providing a new way to enhance the damage effectiveness of the warhead.
\end{abstract}

\section{Introduction}

Discrete rod is a common warhead penetrator[1-2]. The existing structure of discrete rod usually is cross-section long-rod, also existing the pre-formed discrete rod, getting by grooving in the shell structure. Adjust the equipment by the shape of detonation wave, discrete rod can relatively obtain an accord dispersing velocity at the direction of length, forming an end-to-end cutting loop in certain feature distance, so has a strong ability of destruction to the thin-walled structure and skin structure.

When discrete rod hitting the target, its length direction usually parallels the target, discrete rod has a maximum ability of incision at this moment, the disadvantage of this strike model is penetration capability to the big wall-thickness targets relatively weaker. To further improving the penetration capability of discrete rod, Ning Huijun et al. come up with a variable cross-section discrete rod which has a character of both rotating and turning[3], the radius of circular cross-section at length direction of this discrete rod structure increases linearly, presenting a elongated frustum appearance. By the driving of detonation, discrete rod can rotate, in specific kill radius, the length direction of discrete rod directing to the radial direction of the warhead, the penetration capability is relatively strong when hitting the target in this time.

put forward a structure of non-circular cross-section discrete rod, the difference with the above-mentioned discrete rod which its cross-section radius is increasing gradually, is the discrete rod structure putted forward by this thesis is a banding structure with a certain wall-thickness gradient, this structure will radial disperse and rotate simultaneously that driving by sliding detonation, can be able to vertically hitting the target at the length direction, enhancing the ability of penetration to the thick-walled target notably.

\section{Discrete rod and its charge structure}

As figure1(a) shows, non-circular cross-section discrete rod presents stripe, the head and tail are in same width, but the wall-thickness presents linearly increasing along the length direction, compared with cross-section discrete rod which radius is increasing gradually, the former sectional area is linear growth, the latter is index growth.

As figure 1(b) shows, discrete rods are cycle configuration outside the surface of columnar charge, the end with less wall-thickness of discrete rods close to the initiation end of charge. Charge is initiating with center point initiation at the end, forming sphere detonation and spreading to the direction of length, as the way of sliding detonation to drive the discrete rods dispersing at the direction of radial. 


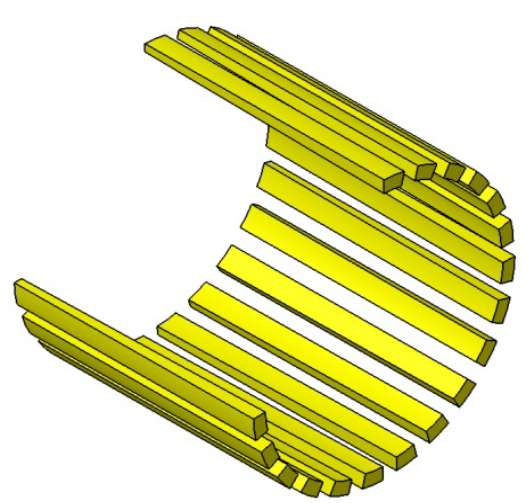

(a) The structure of discrete rods

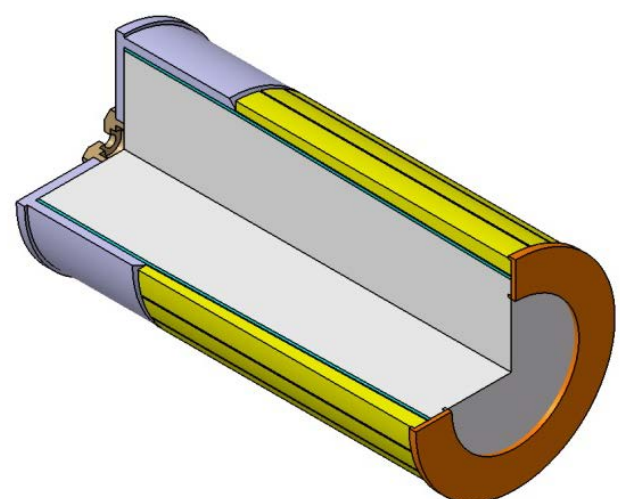

(b) Charge configuration integration of discrete rods

Fig. 1 Non-circular cross-section discrete rods and its pattern of charge integration

As figure 1(b) shows, discrete rods are cycle configuration outside the surface of columnar charge, the end with less wall-thickness of discrete rods close to the initiation end of charge. Charge is initiating with center point initiation at the end, forming sphere detonation and spreading to the direction of length, as the way of sliding detonation to drive the discrete rods dispersing at the direction of radial.

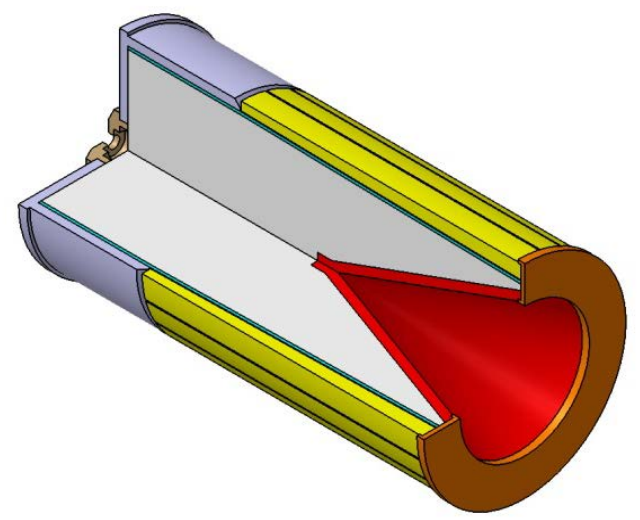

Fig. 2 Cavity structure of the charge fore-end

For the velocity of one infinitesimal element acquired at length direction of the discrete rods is related to the its charge thickness, doing further adjustment on the basis of the structure that showed in figure1(b), making fore-end charge into cavity structure to decrease the racial thickness of this point of charge, as figure 2 shows, the aim is to decrease the local velocity of discrete rods, impelling discrete rods rotating quickly.

\section{Numerical simulation}

simulation model Use hydrodynamic code AUTODYN, analyzing the driving process of discrete rods, simulation model is showed in figure3.

The diameter of the columnar charge as the example is $64 \mathrm{~mm}$, length is $160 \mathrm{~mm}$, Discrete rod length is $100 \mathrm{~mm}$, one end thickness is $3 \mathrm{~mm}$, another is $5 \mathrm{~mm}$, number of discrete rod that distributed in charge circumferential are 24.

In simulation model, charge is B-explosive; material of discrete rod is pure iron, one discrete rod is $25 \mathrm{~g}$. 


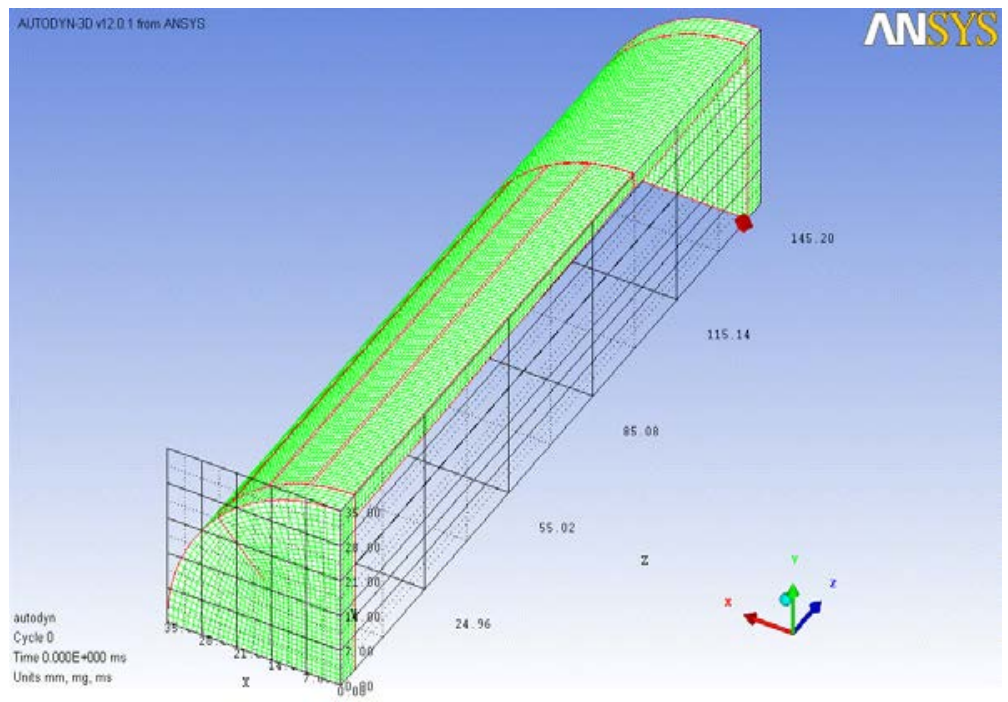

Fig. 3 Discrete rods end-shell \&1/8 shell model

Discrete rod rotate model analysis Driving by sliding detonation, discrete rods present radial dispersing and rotating Simulate, its dispersing process is showed by figure4.

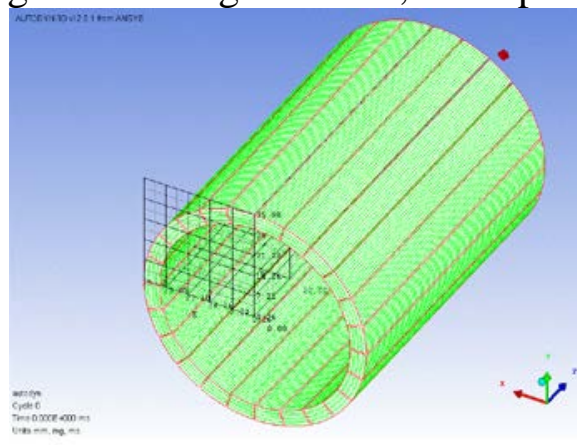

(a) 0 us

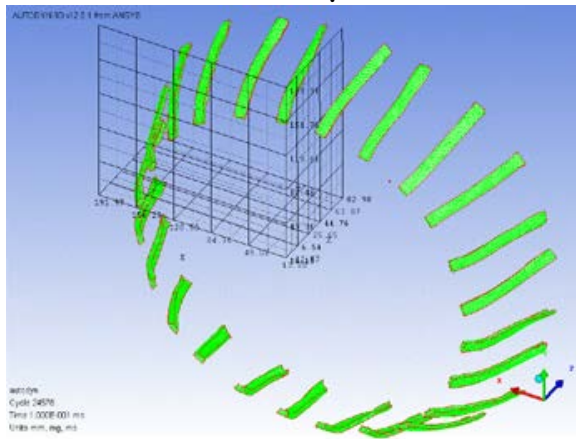

(c) $100 \mu \mathrm{s}$

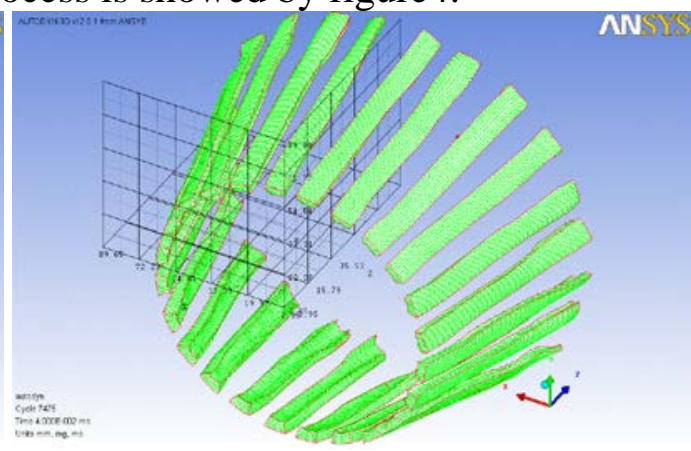

(b) $40 \mu \mathrm{s}$
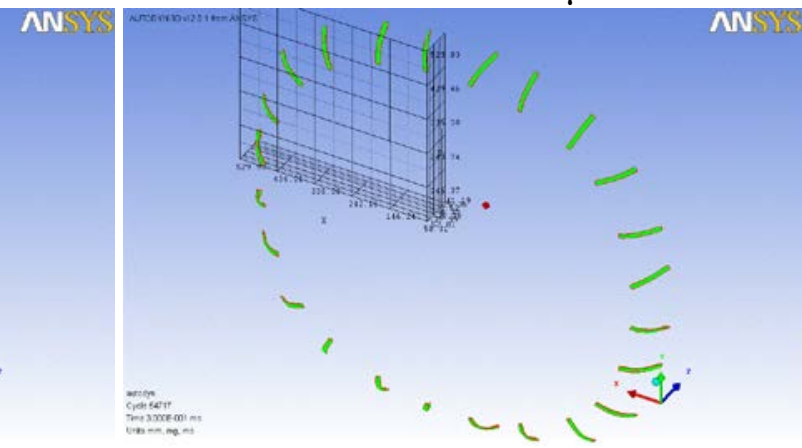

(d) $300 \mu \mathrm{s}$

Fig. 4 Dispersing status of discrete rods in different times (whole model)

As picture shows, 300us after the charge initiation, discrete rods essential spread completely, length direction at now is accord with the radial direction of the charge, the distance between strip and charge axis is about $490 \mathrm{~mm}$, rotate angular-velocity is about $5.2 \mathrm{rad} / \mathrm{ms}$.

Because sliding detonation driving the discrete rods radial dispersing and giving a axial velocity simultaneously, so when length direction of discrete rods is directing to the radial direction of the charge, in the relative front of the charge, its radial velocity component and front velocity component is $1620 \mathrm{~m} / \mathrm{s}$ and $183 \mathrm{~ms}$ separately.

In status of one charge fore-end without cavity structure and another charge fore-end with cavity structure, the deformation contrasts of discrete rod was showed in figure 5. 


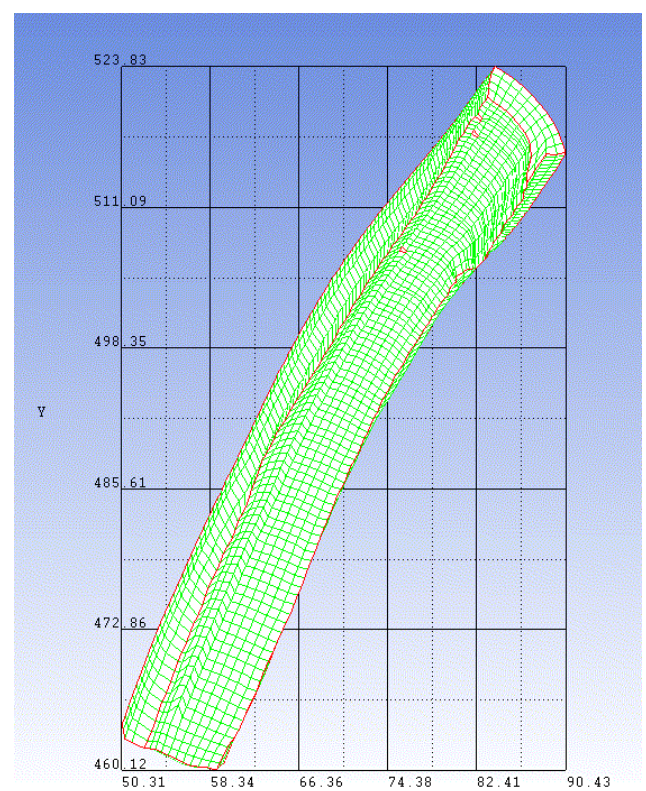

(a) rods status-charge without cavity

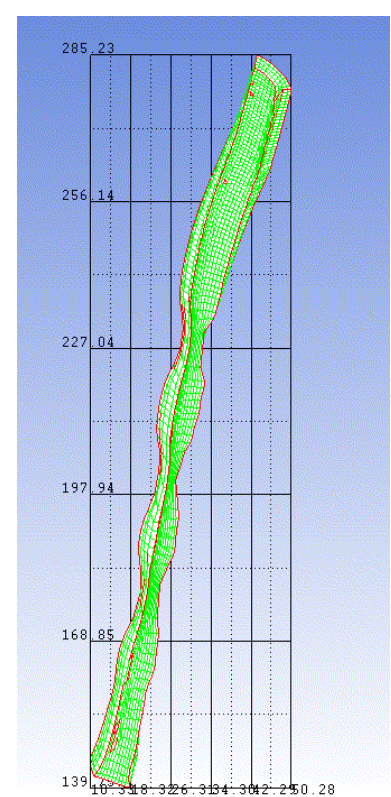

(b) rods status-charge with cavity

Fig. 5 Stretching status of the discrete rod

When charge fore-end with no-cavity structure, the length of discrete rods through stretching were is 1:2.1 to the original length, but wall-thickness keep linear variation essentially. When charge fore-end exists cavity structure, discrete rod was elongated, and it's cross-section shape don't keep linear variation any more, the reason is cavity charge structure leads end loading-wave, the overlyingzone of loading-wave leads discrete rod cross-section to appear local neckdown, discrete rod may fracture.

\section{Summary}

This thesis takenumerical simulation to research the rotate status of varaiable wall-thickness non-circular cross-section discrete rod which is driving by detonation, also analysed the rotat eprocess and the shape of discrete rodunder the influence ofcharge cavity structure.

The advantage of non-circular cross-section discrete rod versus to equal-section discrete rod is, high duty ratio at charge surface、 high driving velocity of discrete rod, high energy utilization efficiency of charge.

The wall-thickness ofnon-circular cross-section discrete rodincreases linearly along the length direction, can disperse that is driving by the charge sliding detonation wave and rotate simultaneously, characteristic radius is relatively less in addition.

For existing the velocity difference at length direction, discrete rods present notable stretching deformation, local necking is occurring under the influence of complex unload-wave, may lead discrete rods to tensile failure.

\section{References}

[1] GAO Shuping, WANG Zhijun, SUN Hua. Influence of structure parameters on performance of discrete rod warhead. ORDNANCE MATERIAL SCIENCE AND ENGINEERING. 2013, 36(3): 71-74.

[2] LV Hongchao, ZHANG Xinwei. Design Methods of Controllable Discrete Rod Warhead. AERO WEAPONRY. $2011:$ 36-38.

[3] NING Huijun, WANG Hao, RUAN Wenjun. Technical research on the design of variable Cross-section rod warhead. ACTA A R MAMENTA R II. 2014, 35(7): 953-959. 